\title{
El reto de los directivos docentes: desarrollo integral a través de las prácticas educativas de la IE Román Gómez
}

\author{
The challenge of teaching managers: integral performance through \\ educative experiences at IE Román Gómez
O desafio da gestão de professores: desenvolvimento integral através das práticas educativas de IE Gomez de Roman

\author{
John Jairo Díaz Muñoz ${ }^{2}$ \\ David Alberto Londoño-Vásquez ${ }^{3}$
}

Recibido: 15.01.2017 - Arbitrado: 16.02.2017 - Aprobado: 14.03.2017

\begin{abstract}
Resumen
Este artículo se desprende del trabajo de investigación realizado en la Institución Educativa Román Gómez de Marinilla (Antioquia) entre el año 2011 y 2013, en el cual se buscó establecer el papel de los directivos docentes en la Institución Educativa y su influencia en el proceso de enseñanza-aprendizaje en relación a las estudiantes de la media vocacional que les permitió destacarse en el medio al realizar las prácticas empresariales. Esta investigación fue cualitativa, hermenéutico-comprensiva. Las técnicas de recolección de información fueron la observación directa en el aula, la entrevista a profundidad a dos directivos y el taller pedagógico a 15 estudiantes. Para el análisis se establecieron como categorías: la educación y la escuela como elementos dinamizadores de la sociedad, el desarrollo humano como meta de la educación, PEI como herramienta de gestión educativa y liderazgo pedagógico. En cuanto a los resultados se pudo colegir que la función y el liderazgo de los directivos docentes ha sido fundamental para el logro de los objetivos propuestos en el componente teleológico del PEI, lo que permitió potenciar la formación integral de los estudiantes, para aprehender los elementos fundamentales de la cultura.
\end{abstract}

Palabras clave: directivos docentes, escuela, desarrollo humano, Proyecto Educativo Institucional, liderazgo.

\footnotetext{
${ }^{1}$ La investigación "Descripción de las prácticas educativas en el grado décimo de la IE Román Gómez: Una relación entre el modelo pedagógico institucional y las experiencias académicas de las estudiantes” se realizó para optar al título de Magíster en Educación y Desarrollo Humano en la Universidad de Manizales-Cinde. Esta tesis fue aprobada a finales de 2014.

${ }^{2}$ Docente IE José Celestino Mutis, Licenciado en Matemáticas, Municipio de Apartadó, Magíster en Educación y Desarrollo, johnjdiazm@gmail.com

3 Doctor en Ciencias Sociales, Niñez y Juventud de la Universidad de Manizales-Cinde, docente tiempo completo de la Institución Universitaria de Envigado. Researcher ID: F-8907-2013. ORCID: 0000-0003-11107930,dalondono@correo.iue.edu.co
}

Citación del artículo: Díaz, J., Londoño-Vásquez, D. (2017). El reto de los directivos docentes: desarrollo integral a través de las prácticas educativas de la IE Román Gómez. Revista Katharsis, N 23, enero-julio 2017, pp.109-128, Disponible en http://revistas.iue.edu.co/index.php/katharsis 


\begin{abstract}
This paper is part of the research work related to the Román Gómez Educational Institution in Marinilla (Antioquia) between 2011 and 2013, in whose objective was to establish teacher managers' roles in an educational institution and its influence in the high school students' teaching-learning process that facilitated them to be stood out in their internship. Some relations were found: besides roles and influences, some challenges tracked by teacher managers in order to invigorate the scholar process. This research was qualitative, hermeneutic-comprehensive. The collection techniques were the direct observation in the classroom, a deep interview to two directive personnel and a pedagogical workshop to 15 students. In the analysis, the categories proposed were education and school as dynamic elements of the society, the human development as the education goal, PEI as an educative management tool and pedagogical leadership. Regarding the results, it was possible to infer that function and leadership of teacher directives have been fundamental for the achievement of the proposed objectives in PEI's teleological component, allowing powering the students' integral formation to apprehend the main cultural elements.
\end{abstract}

Key words: teacher managers, school, human development, Institutional Education Project, leadership.

\title{
Resumo
}

Este artigo mostra o trabalho de pesquisa na Instituição de Ensino Román Gómez de Marinilla (Antioquia) entre 2011 e 2013, cujo objectivo é estabelecer o papel dos administradores escolares na instituição educacional e sua influência sobre o processo de ensino e aprendizagem em relação aos estudantes de meio profissional que lhes permitiu ficar no meio ao fazer práticas de negócios. Esta pesquisa foi qualitativa entendimento hermenêutico. As técnicas de coleta de dados foram a observação direta sala de aula, entrevistas em profundidade dois executivos e oficina educativa para 15 alunos. Para a análise foram estabelecidos como categorias: educação e escola como elementos dinâmicos da sociedade, o desenvolvimento humano como um objetivo da educação, PEI como uma ferramenta para a gestão da educação e liderança pedagógica. Quanto aos resultados, podese inferir que o papel ea liderança dos gestores professores tem sido fundamental para a consecução dos objetivos propostos no componente teleológica do PEI, o que permitiu melhorar a formação integral dos alunos para compreender os elementos cultura fundamental.

Palavras-chave: os administradores da escola, escola, desenvolvimento humano, Institucional Projecto Educativo, liderança.

Citación del artículo: Díaz, J., Londoño-Vásquez, D. (2017). El reto de los directivos docentes: desarrollo integral a través de las prácticas educativas de la IE Román Gómez. Revista Katharsis, N 23, enero-julio 2017, pp.109-128, Disponible en http://revistas.iue.edu.co/index.php/katharsis 


\section{Introducción}

En la Institución Educativa Román Gómez, ubicada en el municipio de Marinilla, departamento de Antioquia, entre los años 2011 y 2013, se realizó la investigación Descripción de las prácticas educativas de las estudiantes del grado décimo: una relación entre el modelo pedagógico institucional y las experiencias académicas de las estudiantes, en la cual se exploraron varias posibilidades, con respecto a las miradas que se pueden dar a los componentes de la comunidad educativa. Esto permitió explorar las posibles relaciones entre el liderazgo de los directivos, las prácticas educativas de los docentes y las actividades de los estudiantes.

En el ámbito municipal de Marinilla, la IE Román Gómez se ha logrado posicionar social y educativamente, por las prácticas empresariales de las estudiantes de la media vocacional. En este estudio se pretendió establecer las posibles relaciones entre el liderazgo de los directivos docentes y el cumplimiento de los objetivos propuestos en el Proyecto Educativo Institucional (PEI), describiendo las prácticas que se realizan al interior de la IE, que promueven el desarrollo de los conocimientos, las cuales están presentes en las prácticas empresariales de las estudiantes en mención.

Esto hace una referencia al modelo pedagógico, que es gestionado por los directivos docentes y que les ha permitido a las estudiantes al realizar las prácticas, actuar de manera destacada en el ámbito social. Aspecto que vale la pena revisarse; puesto que, tiene una cercana incidencia con las prácticas educativas, la implementación del plan de estudios y las estrategias didácticas, lo cual se conecta con los componentes teleológicos del PEI (Builes, Díaz y Ramírez, 2014).

\section{Metodología}

La investigación utilizada es de tipo cualitativo con un enfoque epistemológico de corte hermenéutico-comprensivo (Londoño \& Castañeda, 2011; Naupas et al., 2014) que permitió reflexionar frente a los diversos componentes que tiene el proceso educativo, específicamente en lo que tiene que ver con las actividades de los directivos docentes en relación al modelo pedagógico de la IE Román Gómez, además del análisis de las prácticas educativas docentes y las experiencias de las estudiantes.

Con el objetivo de recolectar la información, como se expresa en el informe técnico del estudio realizado Builes, Díaz \& Ramírez (2014) se utilizaron varias técnicas, como la observación directa en el aula, la entrevista a profundidad y el taller pedagógico (Denzin \& Lincoln, 2015):

- Observación en el aula: presencia de algunas de las actividades desarrolladas por los docentes hacía los estudiantes.

- Entrevista a profundidad: elaboración de un derrotero de preguntas a docentes, directivos docentes y estudiantes, el cual se complementó de acuerdo a la necesidad temática a desarrollar y, su posterior, puesta en escena.

Citación del artículo: Díaz, J., Londoño-Vásquez, D. (2017). El reto de los directivos docentes: desarrollo integral a través de las prácticas educativas de la IE Román Gómez. Revista Katharsis, N 23, enero-julio 2017, pp.109-128, Disponible en http://revistas.iue.edu.co/index.php/katharsis 
- Taller pedagógico: resolución de una situación problema por un grupo de 15 estudiantes con elementos, con los cuales debían proporcionar una utilización distinta a la asignada.

En la búsqueda de la respuesta a este planteamiento se trazó una estrategia: establecer el estado de construcción de los documentos rectores de la Institución, en este caso, el PEI, la cual debía contener los cuatros componentes clave, donde se definieran las acciones que debía realizar en cada uno de ellos, hacia el logro de las metas, fines y propósitos. Se utilizó para ello información de primera mano suministrada por directivos docentes de la IE Román Gómez.

Posteriormente, se buscó información que corroborara la aplicación de los lineamientos trazados. Para ello, se aplicaron entrevistas a profundidad, a un directivo docente, a dos docentes y a un exalumno, y se hizo una visita de campo donde se observaron y registraron diversas facetas de la dinámica escolar. Luego se seleccionó una muestra de quince estudiantes del grado décimo de la modalidad de comercio, con quienes se efectuó un taller pedagógico donde resolvieron un problema no común, del cual se hicieron varios vídeos. Finalmente, se obtuvo la información y se procedió a su análisis a través de una matriz elaborada para el efecto; cruzando la información a partir de las categorías encontradas.

\section{Principales hallazgos del estudio}

\section{Algunas categorías de la docencia directiva en la IE Román Gómez}

Para poder dinamizar los procesos educativos, los directivos docentes consideran una serie de criterios referentes a la enseñanza, que afectan el aprendizaje, en las diferentes disciplinas, en lo relativo a la formación de los estudiantes, además, de las relaciones con los otros componentes de la comunidad educativa, porque desde su gestión como líderes pueden influir directamente en los resultados escolares (López, García \& Slater, 2007).

La escuela, término genérico, tiene funciones complejas y recibe influencias de la sociedad, la cultura y el contexto, además, de fenómenos como la globalización que impactan su funcionamiento. En su interior, el directivo docente como adalid, debe construir un proyecto que le sirva de guía y que le permita realizar una serie de acciones hacia la consecución de unos objetivos educativos y sociales claramente trazados (Cruz, 2013); es decir, debe implementar y permitir la apropiación, por parte de los estamentos que integran la comunidad educativa del PEI, dirigiendo sus acciones hacia los componentes de esta carta de navegación (Roldan, 2011). Son cuatro estos componentes a saber: a) el teleológico que se relaciona con la misión y la visión; b) el académico, que hace referencia al plan de estudios; c) el comunitario que tiene que ver con la contextualización y, por último, d) el directivo que tiene que ver con los criterios de la acción gerencial del directivo.

Estos cuatro componentes deben pensarse de una manera integral que faciliten el desarrollo de las actividades tanto académicas como administrativas correspondientes al proceso de formación presentes en una IE, (re)conectando, (re)construyendo y amalgamando los componentes entre sí. Especialmente, si se entiende que la escuela para su funcionamiento

Citación del artículo: Díaz, J., Londoño-Vásquez, D. (2017). El reto de los directivos docentes: desarrollo integral a través de las prácticas educativas de la IE Román Gómez. Revista Katharsis, $\mathrm{N}$ 23, enero-julio 2017, pp.109-128, Disponible en http://revistas.iue.edu.co/index.php/katharsis 
tiene la característica de una organización en la cual el rector (o su equivalente) es el líder tanto administrativo como académico:

El éxito o el fracaso por mejorar la educación, está centrado en la enseñanza, y que ésta dependerá, en gran parte, de la claridad de las intenciones que se haya trazado la institución, de la generación de compromisos con el cuerpo docente, en el sentido de adecuar las metodologías, contenidos, estrategias didácticas, desde cada una de las disciplinas y en general todo el currículo, adaptándolos a las condiciones específicas de esa comunidad (Concha, 2007, p.133).

En cabeza de dicho directivo, recae la responsabilidad, no solo de dinamizar este proceso, sino de direccionar estos asuntos de manera objetiva y alcanzar las metas propuestas en el PEI, para lo cual, además, será fundamental "el trabajo en equipo, el uso de canales de comunicación efectivos y el propiciar acuerdos y acciones comunes, de manera que él sea un factor diferencial, hacia la obtención de altos niveles de logro en la escuela" (López, García \& Slater, 2007, p.141). Este es uno de los logros importantes de la IE Román Gómez, tal como se constató en la visita de campo y en los testimonios de los estudiantes y docentes. ${ }^{4}$ En la entrevista el docente 1 expresa:

[...] un conjunto de todo, de todo lo que genera esa motivación en los estudiantes; no sólo la formación que es innegable que es muy buena, para mí pues el colegio siempre ha tenido un corte bastante estructurado frente lo académico; es la formación articulada en valores, en tener muy en cuenta a la humanidad, pues como esa parte humana de las estudiantes (E 6).

En suma, se puede decir que la cristalización de los objetivos establecidos en el PEI, la dinámica que generen, las relaciones que establezcan, todo este conjunto, impacta la enseñanza y el aprendizaje, que constituyen los retos que los directivos docentes tienen para la formación de personas capaces de vivir en sociedad con competencias laborales, sociales y ciudadanas y puedan desenvolverse en un mundo que privilegia los conocimientos.

Así mismo, para su accionar, el directivo docente como líder educativo ${ }^{5}$ debe generar una dinámica que dependerá de la utilización de unos conceptos bases sobre los cuales edifique su misión y que lo guiarán para la obtención de los logros trazados en la IE que él dirige. Estos pilares se exponen a continuación.

\section{$\underline{\text { La educación y la escuela como elementos dinamizadores de la sociedad }}$}

El primer concepto que debe tener claro un directivo docente es el de educación, pues sobre él debe instalar su acción. Son los fundamentos por donde caminará en la consecución de los objetivos y propósitos que se ha planteado la IE. Desde la etimología educar quiere decir 'hacer aflorar', en otras palabras, es hacer salir, desde adentro de la persona. En este sentido, Ocampo (2010) plantea que:

\footnotetext{
${ }^{4}$ Esta información se encuentra ampliada en Builes (2016).

5 Término propuesto por Sepúlveda \& Lagomarsino (2007).
}

Citación del artículo: Díaz, J., Londoño-Vásquez, D. (2017). El reto de los directivos docentes: desarrollo integral a través de las prácticas educativas de la IE Román Gómez. Revista Katharsis, N 23, enero-julio 2017, pp.109-128, Disponible en http://revistas.iue.edu.co/index.php/katharsis 
Todo proceso educativo implica una intención de 'hacer aflorar' y de hacerlo de manera sistemática para lograr tal propósito y puesto que este proceso depende de la manera como la sociedad a la que pertenece concibe al ser humano, entendemos por teoría educativa el conocimiento formalizado sobre los factores que inciden en la permanente constitución de lo humano mediado por procesos educativos institucionales que se caracterizan por ser legitimados, intencionales y sistemáticos y que, por ende, permite comprender dichos procesos y darles validez (p.82).

Se trata de desarrollar las dimensiones que constituyen a cada persona, para eso la escuela se traza unos propósitos que consigna en el componente teleológico del PEI, así entonces, la educación es un proceso intencional. Además, la educación tiene una función esencial que consiste básicamente en el desarrollo continuo de las personas y de las sociedades, donde se procure su desarrollo integral de la persona, de tal manera que, al actuar en el medio social, logre la transformación que elimine la exclusión, la pobreza, las incomprensiones y la violencia (Delors, 1996). Se exige entonces que el estudiante asuma la construcción del conocimiento no solo en el paso por la escuela, sino que, por el contrario, sea allí donde construya las herramientas cognitivas necesarias para poder aprender durante la vida.

En síntesis, la educación es un proceso intencional, de contenido humano, que permite que las personas se adapten y actúen en distintos contextos (Builes, 2016). Por tanto, para el desarrollo del proceso educativo se requiere de un espacio especial, socialmente determinado con potencialidades interactivas, al cual se le conoce como escuela. Es por ello que Vizcaíno (2003) señala que en relación a la escuela "se propone, la utilización del conocimiento y la experiencia más depurados. La potenciación del sujeto” (p.129); es decir, el encuentro del estudiante -sujeto constructor de conocimiento- consigo mismo que proponen Londoño y Ospina (2016), en relación a la escuela: “... nos permite participar en el mundo de diferentes formas, puesto que, podemos dilucidar los elementos ideológicos, demagógicos y retóricos que buscan prolongar o afianzar el poder los opresores, poder que sostienen hasta que los oprimidos no se emancipen" (p.253).

Al respecto Froebel (1918, citado por Arias, 2010) expresa el objetivo de la escuela como el "dar a conocer al joven la esencia, el interior de las cosas, y la relación que tienen entre sí, con el hombre y con el alumno, a fin de mostrarle el principio vivificador de todas las cosas..." (p.10). La escuela, entonces, da pie a la manifestación de multiplicidad de expresiones individuales que construyen el colectivo cultural y que, a su vez, se transforma a sí mismo, la sociedad transforma a sus integrantes y estos, a su vez, la transforman a ella.

Es allí donde se plasman las intencionalidades y se realizan las actividades adecuadas para que haya la apropiación de los elementos de la cultura por parte de las nuevas generaciones, que jalonadas por los directivos docentes puedan cristalizar en la práctica los propósitos de la educación. Por su parte la estudiante 1 plantea que:

En la institución educativa los proyectos de la institución van como al crecimiento y no se limitan sólo a lo intelectual. Se limitan mucho en sí con usted como persona... el desarrollo humano de todos los estudiantes, intentan como un buen ambiente, promueven mucho como el compañerismo, o sea que entre todos interactuemos (E1).

Citación del artículo: Díaz, J., Londoño-Vásquez, D. (2017). El reto de los directivos docentes: desarrollo integral a través de las prácticas educativas de la IE Román Gómez. Revista Katharsis, N 23, enero-julio 2017, pp.109-128, Disponible en http://revistas.iue.edu.co/index.php/katharsis 
En este sentido, López, García \& Slater (2007) expresan que: "La escuela es un espacio complejo, singular, específico donde se refleja la realidad social circundante, hecho que le inyecta particularidades que la diferencian de otros con funciones de transmisión sociocultural y de formación" (p.134). Al respecto, Londoño (2015) señala que: "La sociedad es quien define cómo debe ser el hombre y, a su vez, la función principal de la educación es formar ese tipo de hombre deseado para un lugar y una época específicos, en el menor tiempo posible, pero de forma paulatina" (p.56). Como lo expresa el Directivo 2:

"[...] se ve un ser humano bien adaptado a la sociedad, un ser humano muy competente-pensante, como que cierta inteligencia es como que la tienen, se ha logrado desarrollar" (E5).

En otras palabras, en la IE Román se tiene en cuenta las condiciones, características y particularidades propias del municipio de Marinilla para dar respuesta adecuada a estas necesidades sociales, en lo educativo, a través de un currículo contextualizado y dinamizado por el equipo líder institucional.

Además, es necesario considerar por un lado, la cultura referida a los valores y creencias que dan sentido a las actividades que se realizan en la escuela y, por otro lado, la grupalidad, que se refiere a las relaciones entre los directivos y los docentes con respecto al trabajo escolar. Por último, se requiere considerar la motivación como elemento transversal asociada al trabajo en la escuela, que implica un compromiso de los docentes con los aprendizajes de los estudiantes (Fernandez, 2004, citado por Blanco, 2007). Así entonces, el clima escolar propiciado por los directivos adquiere mayor trascendencia en la dinámica escolar para el logro de los objetivos trazados.

Al respecto, el sociólogo francés Durkheim (2000[1922]) defiende la idea que la educación es algo social, tanto por sus orígenes, como por sus funciones, por ende, la pedagogía depende de la sociología más que de cualquier otra ciencia. Por ello, “... la motivación a los profesores, la motivación a los estudiantes, el trabajo en equipo y la comunicación efectiva son características de un liderazgo efectivo" (Bravo \& Verdugo, 2007, p.10), se promueven de esta manera procesos heurísticos en el interior de la escuela, lo que redundará en el mejoramiento de la enseñanza-aprendizaje. Con la utilización de estos elementos se indica claramente las posibilidades de convertir la escuela en un espacio donde se construye el conocimiento, se aplica propiciando desarrollo de competencias por parte de los estudiantes y, en consecuencia, se constituya en uno de los ejes de la transformación social. En otras palabras, "(Con respecto a la sociedad y los estudiantes)... en la que no sólo opinen, sino que sean tenidos en cuenta para la toma de decisiones, las cuales al fin y al cabo, los regirá en los años venideros" (Londoño \& Castañeda, 2010, p.10).

El concepto de educación lleva consigo varios actores, uno de ellos es el estudiante, que se constituye en el centro. Para la IE Román Gómez, el educando se percibe, según expresa uno de los entrevistados, como una persona que tiene:

Citación del artículo: Díaz, J., Londoño-Vásquez, D. (2017). El reto de los directivos docentes: desarrollo integral a través de las prácticas educativas de la IE Román Gómez. Revista Katharsis, N 23, enero-julio 2017, pp.109-128, Disponible en http://revistas.iue.edu.co/index.php/katharsis 
"Un profundo respeto de la norma, son respetuosos de todo, son eh... se perfila un ambiente de mucha armonía, gozan en el colegio, ellos se amañan mucho aquí" (E1) ${ }^{6}$

Es decir, se trata de hacer aflorar todas las potencialidades de los jóvenes, a través del conocimiento formalizado, consignado en el PEI y liderado por los directivos (Ocampo, 2010), de tal manera, que adquiera actitudes de respeto por las normas de comportamiento social aplicadas en la escuela, generándose un buen ambiente de aprendizaje permeado por una motivación de los estudiantes hacia el proceso educativo (Sanz \& Rodríguez, 2000).

Además, como lo expresa uno de los directivos entrevistados:

"[...] se ve un ser humano bien adaptado a la sociedad, un ser humano muy competente-pensante, como que cierta inteligencia es como que la tienen, se ha logrado desarrollar" (E5)

Es decir, se privilegia un ser humano competente-pensante, que se ubica y adapta a la sociedad. Ahora, el docente 2 expresa:

"Yo digo que sin el estudiante no habría ningún proceso y que realmente es la persona la cual viene a recibir orientaciones para ayudarles en su crecimiento intelectual" (E6).

En otras palabras, la educación tiene una función social que desde la escuela se imprime, de tal manera que el estudiante sea un transformador de sí mismo y de la sociedad (Delors, 1996), esto implica, además, que el estudiante es centro del proceso educativo, el cual es liderado por los directivos con un norte que es el PEI (Cano, 2008, citado por Carrera \& Marín, 2011).

Por otra parte, la motivación se convierte en una estrategia para que el joven se decida a emprender el camino del conocimiento. Uno de los docentes entrevistados expresa con respecto a la importancia de la motivación que, es:

"[...] un conjunto de todo, de todo lo que genera esa motivación en los estudiantes; no sólo la formación que es innegable que es muy buena, para mí pues el colegio siempre ha tenido un corte bastante estructurado frente lo académico; es la formación articulada en valores, en tener muy en cuenta a la humanidad, pues como esa parte humana de las estudiantes" (E6).

Se manifiesta aquí el efecto de la motivación para el proceso educativo, considerándose al estudiante como un ser humano, como ser social que actúa adecuadamente en este espacio y en esta época (Londoño, 2015), porque se da a conocer el interior de las cosas y las relaciones con él mismo y con la sociedad (Froebel, 1918, citado por Arias, 2010). La

\footnotetext{
${ }^{6}$ En este artículo, se irán incluyendo algunos enunciados tomados de las diferentes entrevistas realizadas en la investigación, cuyas transcripciones se encuentran en Builes, Díaz y Ramírez (2014). Por tanto, con el ánimo de dar un orden y proporcionar claridad comprensiva en el texto, se utilizará la codificación de la entrevista y el número del anexo. Por tanto, E1 significa que es la entrevista que corresponde al anexo 1 de la tesis Builes, Díaz \& Ramírez (2014).
}

Citación del artículo: Díaz, J., Londoño-Vásquez, D. (2017). El reto de los directivos docentes: desarrollo integral a través de las prácticas educativas de la IE Román Gómez. Revista Katharsis, N 23, enero-julio 2017, pp.109-128, Disponible en http://revistas.iue.edu.co/index.php/katharsis 
formación integral del estudiante es uno de los propósitos teleológicos del PEI. Uno de ellos en la entrevista dice:

"Por la misma formación que nos han dado en la institución, como se enfocan tanto como en el trato con la persona, ese respeto, pero no tampoco ese miedo; sino un respeto hacia la autoridad. Me parece que eso es de útil ayuda, pues porque cuando una persona ve que ella me sirve en la empresa, me respeta, compaginamos; es una persona que si no sabe, es atenta, pregunta; me parece que eso les llama mucho, o sea no es tanto que la persona que sepa, sino que quiera, que se le vea ese interés" (E7).

Por tanto, la cultura referida a los valores y la grupalidad, en cuanto a las relaciones entre directivos y docentes, dinamiza el proceso educativo (Fernández, 2004, citado por Blanco, 2007). Por consiguiente, en el trabajo escolar se da pie para el buen trato entre las personas y las instituciones sociales, propiciando así un buen ambiente convivencial, que se constituye en una condición para la formación integral (Bravo \& Verdugo, 2007).

\section{$\underline{\text { El desarrollo humano como meta de la educación }}$}

Hablar de las características de las personas, implica el concepto de desarrollo humano, que, por supuesto, es de orden teleológico para la escuela y para quien la dirija. Para efecto de lo anterior, se toma la definición de desarrollo humano planteada por González (2010): "el desarrollo humano, puede entenderse como el proceso de constitución del sujeto individual y colectivo; un sujeto histórico, cultural y socialmente posicionado" (p.18); es decir, se considera al ser humano en su integridad, en su totalidad, lo que correspondería a su complejidad y sus dimensiones. Por tanto, el proceso educativo en la escuela debe permitir a sus estudiantes desarrollar capacidades, cualidades y talentos de tal manera que cada uno de ellos se responsabilice por su proyecto de vida.

El tema del desarrollo humano no es algo que no se pueda agotar en un autor, y menos en una disciplina $o$ en una situación histórica determinada. Este depende del desenvolvimiento humano, del contexto en que se encuentre ubicado, de las relaciones que se establezcan, es decir, se construirá en la medida del desenvolvimiento de los funcionalismos fundamentales del ser humano (Bazdresch, 1988). Autores como Max Neef, Elizalde \& Hopenhayn (1998), Hanna Arent (2007) y Amartya Sen (2001) ubican al desarrollo humano como el proceso mediante el cual es posible aumentar las opciones para todos los habitantes de un país o región en diversos ámbitos como lo son el educativo, laboral, material, recreativo y cultural, por mencionar algunos.

Al respecto, Max Neef, Elizalde \& Hopenhayn (1998) proponen la perspectiva de las necesidades y satisfactores, la cual plantea que el desarrollo humano son las personas, no las cosas, que si bien se refiere a la parte económica esta no es un satisfactor suficiente y no alcanza a abarcar todas las necesidades humanas.

Por su parte, Hanna Arent (2007) plantea la perspectiva de los derechos que toda persona debe poseer, tales como el derecho de la ciudadanía, es decir, a pertenecer a cualquier estado que se convierten en ciudadanía al ejercerlos, lo que implica deberes o responsabilidades que el Estado reconoce para todos los ciudadanos.

Citación del artículo: Díaz, J., Londoño-Vásquez, D. (2017). El reto de los directivos docentes: desarrollo integral a través de las prácticas educativas de la IE Román Gómez. Revista Katharsis, N 23, enero-julio 2017, pp.109-128, Disponible en http://revistas.iue.edu.co/index.php/katharsis 
La perspectiva de las capacidades y libertades que propone Amartya Sen (2001) se basa en la idea de que el verdadero desarrollo debería ser un proceso de expansión de las libertades reales de que disfrutan los individuos. En otras palabras, "un modelo de desarrollo que tenga en cuenta esta perspectiva necesariamente debe plantearse la eliminación de todas las fuentes de privación de la libertad como pueden serlo la pobreza, la tiranía, la escasez de oportunidades económicas y las privaciones sociales sistemáticas, la deficiencia en los servicios públicos, etc.” (González, 2010, p.8).

Por otra parte, María Teresa Luna (2010) propone la perspectiva de las potencialidades, en la cual se considera que el desarrollo humano está formado por diversas dimensiones tales como: la orgánica - madurativa, la erótico - afectiva, la ético - moral, la política, la lingüístico-comunicativa, la productiva y la lúdico-estética. Acá se podría señalar que la escuela propicia, a través de cada una de las disciplinas del saber, el desarrollo de estas dimensiones, por medio de las distintas estrategias metodológicas de acuerdo también al modelo pedagógico institucional.

Teniendo en cuenta lo anterior, el rol de docentes tiene varias implicaciones como lo expresa el Directivo 1 diciendo que:

"Los profesores que son patrimonio del municipio, enseñan el amor por el municipio, no sólo enseñan los conceptos básicos de x o y materia sino que con su vida y testimonio invitan a los estudiantes a ser felices dentro de lo que ellos son. Motivan a los estudiantes para que construyan un proyecto de vida [...]" (E5).

O sea que, la IE, con su propuesta teleológica, liderada por los directivos, induciendo a los docentes que la apliquen desde el área específica, se promueve elaboración del proyecto de vida personal, que es la base de la formación integral; a través del ejemplo de vida y de la motivación. A su vez el docente 2, desde su labor, expresa que:

"No es un doble trabajo yo creo que es un gran compromiso para que ellos desarrollen competencias y así cuando pasen a los años siguientes pasen muy seguros de haber desarrollado realmente temas o de pronto prácticas que para ellos sean mucho más fáciles adquirirlas en el año siguiente. Los profesores no nos vemos como el profesor que va y dicta su clase y se va; si no que se tiene presente toda esa parte humana de las niñas. Entonces hay un acompañamiento, hay un vínculo en lo afectivo con las niñas" (E6).

Es decir, en la IE, al dinamizar el plan de estudios por los directivos, las prácticas educativas de los docentes, no se limitan a la acción inmediata que se desarrolla en el aula de clase, sino que se proyecta en toda una propuesta de formación integral en el tiempo; propiciando así un buen ambiente de enseñanza-aprendizaje. El estudiante 2 por su parte, plantea que:

"La gran mayoría de veces sí, el profesor sí intenta como que nosotros visualicemos-practiquemos lo que vemos" (E7).

Por otro lado, sigue expresando en la entrevista la Directiva 1 sobre los docentes:

Citación del artículo: Díaz, J., Londoño-Vásquez, D. (2017). El reto de los directivos docentes: desarrollo integral a través de las prácticas educativas de la IE Román Gómez. Revista Katharsis, N 23, enero-julio 2017, pp.109-128, Disponible en http://revistas.iue.edu.co/index.php/katharsis 
"Entonces los motivan a que tengan un proyecto de vida, a que sean exitosos, a que en ambientes zanahorios como los llaman los estudiantes se puede ser muy feliz. Son generadores de ambientes de aprendizaje porque los invitan a la construcción, se utilizan todos los recursos, no se tiene a los estudiantes como encerrados en cada aula como se quiere ahora por ejemplo" (E7).

Es claro como desde las intencionalidades de la escuela en el PEI y lideradas por los directivos, se induce desde diversos ángulos, en el aula y fuera de ella, la elaboración del proyecto de vida en los estudiantes, a través del uso de recursos pedagógicos que proporciona la IE, lo cual facilita el desarrollo de las distintas dimensiones del ser.

Los procesos educativos desarrollan su propia dinámica, que permite aportar al desarrollo humano de los estudiantes, tal como expresa una de ellas (E1):

En la IE, los proyectos de la institución van como al crecimiento y no se limitan sólo a lo intelectual. Se limitan mucho en sí con usted como persona... el desarrollo humano de todos los estudiantes, intentan como un buen ambiente, promueven mucho como el compañerismo, o sea que entre todos interactuemos (E7).

Es decir, se promueve dentro de la escuela por parte de los directivos la realización de actividades en las distintas disciplinas del saber que potencian la enseñanza, y que afectan los resultados escolares (López, García \& Slater, 2007); además, se propicia el crecimiento integral de los estudiantes generando un buen ambiente para el desarrollo humano que les permite interactuar entre ellos (Sanz \& Rodríguez, 2000).

\section{$\underline{\text { PEI como herramienta de gestión educativa }}$}

Para cristalizar estos postulados teleológicos en cuanto a la educación, la escuela y el desarrollo humano, es imperativo que el directivo docente, tenga como referencia el PEI que fue definido por la Ley 115 (1994), como una herramienta a través de la cual la IE, direccionará sus actividades con precisión para el logro de los fines propuestos.

Para Roldan (2011), el PEI debe ser asumido como un "producto estructurado del proceso administrativo que tiene por objeto prever, en términos operativos, el rumbo que tomará la institución" (p.31). Este llama a la comunidad educativa a la reflexión, al análisis como un insumo en la búsqueda de soluciones a los problemas que la afectan, donde el diálogo y la planeación se convierten en ejes articuladores de la acción educativa en su conjunto.

Por tanto, estructurar los procesos en la escuela es dotar de sentido a las actividades que allí se realizan, lo que implica la selección de unos elementos de la cultura, "que se expresan en el currículo como un criterio que permite seleccionar las partes de la cultura, que en la escuela deben ser aprehendidos, por los estudiantes" (Magenzo, 1991, citado por López, 1996, p.18). Este es el sentido de los planes de estudio que para el caso de la IE Román Gómez se han consignado en el PEI y constituyen los propósitos para ser aprehendidos por las estudiantes. Los planes de estudios se basan en modelos pedagógicos, que según Flórez

Citación del artículo: Díaz, J., Londoño-Vásquez, D. (2017). El reto de los directivos docentes: desarrollo integral a través de las prácticas educativas de la IE Román Gómez. Revista Katharsis, $\mathrm{N}$ 23, enero-julio 2017, pp.109-128, Disponible en http://revistas.iue.edu.co/index.php/katharsis 
(1994) "representan formas particulares de interrelación entre los parámetros pedagógicos, una concepción del ser humano específica y de una idea claramente determinada de la sociedad" (p.110).

En otras palabras, las actividades realizadas en la IE Román Gómez dejan una huella en los estudiantes, que se expresa no sólo en los contenidos desarrollados, sino también en las actividades generales que realizan. De Zubiría (1994) expresa que "es en las diversas actividades que se realizan en la escuela donde aparecen las huellas de la concepción pedagógica que se utiliza, tanto en los estudiantes como en los docentes" (p.8). Esto lo corrobora el Directivo 1 en la entrevista, diciendo que una:

"Relación adecuada entre la teoría y la práctica, se evidencia en las prácticas empresariales, en los desfiles, cuando ellos salen a proyectarse al municipio, en las visitas a los museos, en las visitas a las obras de teatro; en donde ellos se ve que la teoría que aprendieron con respecto a los valores, las competencias laborales, las competencias comunicativas y otras, se ven evidenciadas en las prácticas y ya luego en el trasegar de su vida" (E5)

En suma, las prácticas cotidianas del aula de clase, las actividades comunitarias, entre otras, son las manifestaciones del modelo pedagógico, que implica el liderazgo de los directivos docentes en su implementación (Pinto \& Castro, 1999). En la entrevista el directivo 1 expresa:

Esas reflexiones pedagógicas, nosotros las iniciamos, desde que empezamos a inquietarnos acerca del modelo pedagógico, vamos a trabajar el desarrollo del pensamiento, pensamiento creativo, hacer las pruebas por competencias de cada periodo, tratamos de desarrollar mucho el pensamiento ahí, ponerle lógica, se desarrolla la lógica y la comprensión lectora, en el mejoramiento académico" (E5).

Ahora, según Linuesa (2007), las teorías en educación están signadas por un componente intencional y axiológico que las determina en gran medida. Los referentes teóricos a los que se hace referencia se denominan paradigmas, que para el caso del Román Gómez es el cognitivo. En la entrevista, uno de los estudiantes dice:

“... de pronto como en la calidad de los profesores que tenemos, ellos como que siempre son como buscando la manera de explicarnos..., de hacer la forma que nos llegue más fácil la información" (E7)

El PEI se cristaliza en la medida en que en la escuela se realicen prácticas que tengan que ver con alguno de sus componentes. Esto hace que sean intencionales, de tal manera que produzcan un impacto en lo social, laboral y personal. El Directivo 2 en la entrevista expresa:

"Es que yo misma veo y profundizo y profundizo y es un aprendizaje, como que de acuerdo al ambiente, donde se desenvuelven, que ellas saben, tienen que ser excelentes ciudadanas, excelentes trabajadoras, como amas de casa" (E5).

Citación del artículo: Díaz, J., Londoño-Vásquez, D. (2017). El reto de los directivos docentes: desarrollo integral a través de las prácticas educativas de la IE Román Gómez. Revista Katharsis, N 23, enero-julio 2017, pp.109-128, Disponible en http://revistas.iue.edu.co/index.php/katharsis 
Es decir, aquí no se trata de agrupación de circunstancias al azar, más bien es una acción intencionada de los directivos con referencia a los postulados del PEI (Bolívar, 2009). En lo operativo, las estrategias didácticas conllevan una intención de relacionar la teoría y la práctica para que surta un efecto en las vivencias de los estudiantes y produzca una transformación en lo social y personal, con unas intenciones y metodologías propias del modelo pedagógico aplicado.

\section{Liderazgo pedagógico: un requerimiento institucional}

Es evidente, como lo expresa Bolívar (2009), que "este proceso de desarrollo de los estudiantes no se da por una simple agrupación de circunstancias, sino por una acción directiva docente intencional que, al actuar como catalizador, promociona y gestiona el proceso de enseñanza-aprendizaje" (p.2). La escuela debe propiciar la elaboración de los postulados de orden teleológico, para luego ser dinamizados bajo la orientación de los directivos docentes hacia el logro de los propósitos y metas trazados.

Una dirección centrada en la enseñanza y el aprendizaje implica actividades como supervisión de los procesos tanto en los espacios comunes de la escuela como en el aula de clase, es decir, una relación de nuevo tipo que permita interacciones entre los distintos componentes de la comunidad educativa, amplios y profundos, en donde la motivación entre docentes y estudiantes sea un elemento integrador que dinamice el proceso y lleve en última instancia hacia el desarrollo integral de los estudiantes.

Así, entonces, se requiere liderazgo pedagógico de carácter transformador, que supere las prácticas de orden burocrático, tecnocrático o autoritario, en donde se permitan y se tengan en cuenta las acciones de los docentes, la metodología de enseñanza, así como también las inquietudes de los estudiantes o la manera como estos aprenden, este es el caso de las instituciones educativas que están en el nivel muy superior según las pruebas censales en Colombia (Icfes, 2011).

Adicional a lo anterior, la función del directivo docente, como lo expresan Sepúlveda \& Lagomarsino (2007), "es animar, motivar, facilitar desempeños y promover actividades, actitudes y conductas organizacionales para el logro de los objetivos establecidos por la institución" (p.153). Se trata de un apoyo a los trabajos que realiza el docente en el aula y a las demás funciones educativas de la escuela, en donde exista pertinencia con la realidad y se muestre una verdadera coordinación entre lo financiero y lo humano. Una buena gestión educativa, por tanto, debe incluir: claridad en los objetivos, planificación, participación de la comunidad, innovación, liderazgo y relaciones adecuadas con el contexto que permitan obtener apoyo externo, que se muestre además una capacidad de utilizar la oferta pública y se pueda generar así una acción centrífuga de afuera hacia adentro de la escuela.

Cuando un líder toma como objetivo el trabajo en la escuela, esto es de una trascendencia muy grande en los destinos académicos de la institución porque, como expresa Cayulef (2007): "Un directivo docente es un líder que se convierte en un factor de eficacia escolar" (p.144). En otras palabras, su objetivo de trabajo es la escuela sobre la cual ejerce toda su capacidad cognitiva y social. Se puede decir, entonces, que los lideres eficaces, son: organizados y trabajan duro, se relacionan bien con la comunidad, dirigen a través del ejemplo, son flexibles, dialogantes y prefieren comprometer a los docentes en las decisiones

Citación del artículo: Díaz, J., Londoño-Vásquez, D. (2017). El reto de los directivos docentes: desarrollo integral a través de las prácticas educativas de la IE Román Gómez. Revista Katharsis, N 23, enero-julio 2017, pp.109-128, Disponible en http://revistas.iue.edu.co/index.php/katharsis 
que imponerlas; establecen relaciones de amistad pero son claros en la autoridad; les preocupa más el problema que los culpables, y sus resultados tienen que ver con una relación adecuada con el trabajo y las relaciones armoniosas con la escuela, con lo cual crean sentido de pertenencia por ella, motivan y promueven valores culturales sociales y ciudadanos (López, García \& Slater, 2007; Bravo \& Verdugo, 2007).

Es por esto que una dirección centrada en la enseñanza y el aprendizaje promueve dentro de la escuela actitudes, relaciones y comportamientos organizacionales que permean la dinámica escolar hacia el logro de los objetivos (Sepúlveda \& Lagomarsino 2007) en este sentido, el directivo 2 expresa respecto a los docentes que:

"Ellos interactúan enseñando con lo que son, con su ejemplo de vida, la gran mayoría son maestros de muy buena calidad, muy idóneos y que su vida en el pueblo no dan nada qué desear para los estudiantes" (...) y continúa "es generador de un clima convivencial adecuado con los estudiantes, los directivos y los padres... el estudiante deja ver que allí hay un clima institucional agradable, un clima en el cual que se aprende libremente, sin tensiones" (E5).

Se infiere como el docente educa con el ejemplo, desde lo personal hasta lo profesional pero es el directivo el que facilita las labores y promueve desempeños y actividades pedagógicas, además de actitudes se genera un buen ambiente convivencial (Sepúlveda \& Lagomarsino, 2007).

De igual forma, la Directivo 1 expresa que:

"Los maestros son motivadores, son provocativos y provocadores de realidades, de aprendizaje; en cada encuentro de saberes los estudiantes siempre aprovechan esa posibilidad que el maestro les ofrece mediante la construcción de cualquier cosa significativa para ellos... Empezaron con los proyectos en $9^{\circ}$, los inició, un proyecto de investigación, que articula todas las áreas en un... en algo, en lo que los estudiantes se ponen a pensar... y todas las áreas aportan con su conocimiento a ese proyecto" (E5).

En otras palabras, la motivación, se convierte en un elemento transversal que permea el proceso de aprendizaje y facilita el trabajo de construcción de los conocimientos por parte de los estudiantes. Otros sentidos de favorabilidad para el desarrollo de las actividades planeadas, se generan desde la dirección como la diversidad de actividades y los proyectos que desde cada una de las disciplinas aportan a la formación de los estudiantes.

\section{Sistematización de los principales hallazgos}

Con relación a los directivos docentes, para el análisis de la información, se establecieron cuatro categorías, que atañen al modelo pedagógico y su implementación en la IE Román Gómez. Los testimonios tanto de los directivos, docentes y estudiantes fueron explicados desde un presupuesto teórico, y luego se triangularon (Denzin \& Lincoln, 2015)

Citación del artículo: Díaz, J., Londoño-Vásquez, D. (2017). El reto de los directivos docentes: desarrollo integral a través de las prácticas educativas de la IE Román Gómez. Revista Katharsis, N 23, enero-julio 2017, pp.109-128, Disponible en http://revistas.iue.edu.co/index.php/katharsis 
las categorías para obtener conclusiones relacionadas con la participación de los directivos docentes. tabla:

En suma, los hallazgos en las categorías analizadas se pueden resumir en la siguiente

Tabla 1

Hallazgos en relación a la gestión de los directivos docentes.

\begin{tabular}{|c|c|}
\hline Item & Hallazgos \\
\hline $\begin{array}{l}\text { La educación y la } \\
\text { escuela }\end{array}$ & $\begin{array}{l}\text { - Que los estudiantes: } \\
\circ \text { muestren un desarrollo de competencias de acuerdo } \\
\text { a los posibles ambientes de desempeño, en lo social } \\
\text { y personal y que estas sean parte de la formación } \\
\text { integral } \\
\text { - Que los docentes: } \\
\circ \text { implementan a través de las prácticas educativas } \\
\text { conllevan una intención que relaciona la teoría y la } \\
\text { práctica, leyendo las necesidades del contexto y los } \\
\text { propósitos de la institución }\end{array}$ \\
\hline Desarrollo humano & $\begin{array}{l}\text { - Que los estudiantes: } \\
\circ \text { que utilicen el diálogo, que estén motivados y que } \\
\text { además tengan una adecuada formación axiológica. } \\
\circ \text { Que desarrollen cualidades humanas y de } \\
\text { pensamiento que les permitan adaptase a la sociedad } \\
\text { dinámica actual } \\
\text { - Los docentes referencian su accionar en el tiempo hacia la } \\
\text { formación integral del estudiante ubicado en un contexto, }\end{array}$ \\
\hline PEI & $\begin{array}{l}\text { - Se trabaja intencionalmente para: } \\
\circ \text { Formar jóvenes que aprendan a convivir y } \\
\text { desarrollen las competencias ciudadanas, } \\
\circ \text { Que los proyectos y con ellos los contenidos } \\
\text { desarrollen las temáticas básicas en cada una de las } \\
\text { disciplinas del saber y faciliten la construcción de los } \\
\text { conocimientos por parte de las estudiantes y que a su } \\
\text { vez estas temáticas estén formando parte de los } \\
\text { estándares propuestos por el MEN y del plan de } \\
\text { estudios del componente académico del PEI. } \\
\circ \text { usando recursos y estrategias tales como el trabajo en } \\
\text { equipo, la motivación, el diálogo, direcciones de } \\
\text { grupo, salidas de campo, prácticas empresariales } \\
\text { generando un ambiente convivencial y cognitivo } \\
\text { propicio para la construcción del conocimiento } \\
\text { significativo, y la formación integral. }\end{array}$ \\
\hline
\end{tabular}

Citación del artículo: Díaz, J., Londoño-Vásquez, D. (2017). El reto de los directivos docentes: desarrollo integral a través de las prácticas educativas de la IE Román Gómez. Revista Katharsis, $\mathrm{N}$ 23, enero-julio 2017, pp.109-128, Disponible en http://revistas.iue.edu.co/index.php/katharsis 
- Las prácticas educativas docentes y de estudiantes estén enmarcadas en la interinstitucionalidad, a través de los convenios.

Liderazgo pedagógico - Se trabaja intencionalmente para que:

- Los estudiantes con su acción generen un óptimo ambiente de aprendizaje

- Los docentes generan un clima adecuado en las relaciones con los estudiantes y los directivos docentes,

- Desde cada una de las disciplinas se aporte a la formación integral de los estudiantes, utilizando como referencia los estándares y los lineamientos curriculares,

- Los proyectos y los contenidos se convierten en elementos direccionadores que permiten la implementación de variadas estrategias didácticas y todas en su conjunto contribuyen a la formación integral de las estudiantes, así como también al desarrollo de sus competencias, apoyado por la dirección institucional y soportado en los documentos institucionales

Fuente: Elaboración propia

\section{A manera de conclusión}

Se evidencia como el liderazgo de los directivos docentes en la IE Román Gómez, promueven:

\section{En la educación y la escuela}

- Unas prácticas educativas desde las distintas disciplinas, debidamente programadas desde el PEI en sus distintos componentes y que potencializan todo el proceso educativo (López, García \& Slater, 2007) en beneficio de los estudiantes y su crecimiento integral generando un buen ambiente para este desarrollo (Sanz \& Rodríguez, 2000).

- Un conocimiento formalizado a través del plan de estudios, consignado en el componente académico del PEI (Ocampo 2010).

- Unas actitudes convivenciales caracterizadas por el respeto a las normas de comportamiento social y permeadas por una motivación hacia la educación integral (Sanz \& Rodríguez, 2000).

- La participación en las prácticas empresariales y su adecuada valoración, evidencian la función social de la escuela, que transforma a las personas y es un catalizador de

Citación del artículo: Díaz, J., Londoño-Vásquez, D. (2017). El reto de los directivos docentes: desarrollo integral a través de las prácticas educativas de la IE Román Gómez. Revista Katharsis, N 23, enero-julio 2017, pp.109-128, Disponible en http://revistas.iue.edu.co/index.php/katharsis 
transformación social también (Delors, 1996), y que coloca por ende al estudiante en el centro de este proceso (Cano, 2008, citado por Carrera \& Marín, 2011).

- $\quad$ El estudiante se le coloca primero como ser humano y centro del proceso educativo, situado en un contexto que exige vinculación con las organizaciones sociales que allí funcionan (Londoño, 2015).

- $\quad$ Se produce una transmisión de valores culturales y un sentido de la grupalidad en las relaciones entre directivos y docentes que, dinamiza el proceso educativo en su interior (Fernández, 2004, citado por Blanco, 2007).

- Generación de un buen ambiente convivencial a partir de un adecuado clima laboral, condiciones para la implementación de la formación integral en el interior del Román Gómez (Bravo \& Verdugo, 2007).

En el desarrollo humano

- $\quad$ En el Román Gómez con su propuesta teleológica, liderada por los directivos, se promueve elaboración del proyecto de vida personal, que es la base de la formación integral y que conlleva al desenvolvimiento de los funcionalismos del ser humano (Bazdresch, 1988). - Se propicia además el desarrollo del sujeto individual y colectivo ubicado en un contexto histórico (González, 2010).

- El desarrollo de las prácticas educativas, que trae consigo el plan de estudio, aplicadas dentro y fuera del aula, permite el desenvolvimiento de las dimensiones del estudiante (Luna, 2010, citada por González, 2010).

\section{En relación al PEI}

- En el Román Gómez no se trata de agrupación de circunstancias al azar, el reconocimiento social y académico en el contexto del municipio de marinilla, es más bien una acción intencionada de los directivos con referencia a los postulados del PEI (Bolívar, 2009).

- $\quad$ En el PEI se define el modelo pedagógico, que para el Román Gómez es desarrollista, del cual forman parte las estrategias didácticas de los docentes, los proyectos y la participación en actividades del contexto municipal (De Zubiría, 1994).

- $\quad$ En la dinámica de enseñanza aprendizaje se produce una interacción reflexiva entre la teoría y la práctica, que vivenciada por los estudiantes produce un impacto social y personal (Roldan, 2011).

\section{En el liderazgo pedagógico}

- $\quad$ Se evidencia un interés de la dirección en el proceso de enseñanza aprendizaje, favoreciendo la realización de actividades, prácticas y proyectos que han permeado organizacionalmente la dinámica escolar (Sepúlveda \& Lagomarsino, 2007).

- En la favorabilidad organizacional del Román Gómez, se educa desde el ejemplo, produciendo un ambiente académico y convivencial adecuado para la enseñanza y el aprendizaje (Sepúlveda \& Lagomarsino, 2007).

Citación del artículo: Díaz, J., Londoño-Vásquez, D. (2017). El reto de los directivos docentes: desarrollo integral a través de las prácticas educativas de la IE Román Gómez. Revista Katharsis, $\mathrm{N}$ 23, enero-julio 2017, pp.109-128, Disponible en http://revistas.iue.edu.co/index.php/katharsis 
En síntesis, puede decirse que a partir de la investigación realizada en la IE Román Gómez, se puede colegir que la función y el liderazgo de los directivos docentes ha sido fundamental para el logro de los objetivos propuestos en el componente teleológico del PEI. La directriz central de la institución está centrada en el aprendizaje, lo que ha permitido potenciar la formación integral de los estudiantes, para aprehender los elementos fundamentales de la cultura.

Además, se ha generado un sentido de grupalidad que ha permitido la realización de prácticas educativas efectivas, de forma que pueda mirarse no solamente como los resultados altos en las pruebas censales, sino que además se deben tener en cuenta otros factores que inciden en la singularidad de cada IE. De igual forma, un clima escolar académico y convivencial, ha permitido relaciones adecuadas con el contexto municipal.

Finalmente, se evidencia el establecimiento de unos fuertes lazos interinstitucionales para que el ejercicio educativo en el interior de la escuela tenga un sentido de ubicación con el contexto. Al igual que una intención claramente definida en el PEI, para formar personas integrales, en coherencia con las prácticas educativas.

\section{Referencias}

Alzate, F., Jaramillo, A. (2015). La gestión del conocimiento un desafío para las instituciones educativas en Colombia: Emergencias y tensiones desde la teoría del capital intelectual. Revista Gestión de la Educación, 5 (2), 137-150. http://www.facultadeducacion.ucr.ac.cr/revista-facultad/gestion-de-la-educacion

Arias Arteaga, G. (2010). Corrientes pedagógicas contemporáneas. Medellín: CINDE.

Arendt, H. (2007). La condición humana. Barcelona: Paidos.

Bazdresch, J. (1988). ¿Cómo hacer operativa la formación humanista en la universidad? Cuadernos de reflexión universitaria, (14). http://www.worldcat.org/title/cuadernosde-reflexion-universitaria/oclc/863611076

Blanco, E. (2007). Factores sociales asociados a los aprendizajes en la Educación primaria mexicana: un análisis multinivel. REICE-Revista Electrónica Iberoamericana sobre Calidad, Eficacia y Cambio en Educación, 6 (1), 54-88. https://dialnet.unirioja.es/servlet/revista?codigo $=3322$

Bolívar, A. (2009). Una dirección para el aprendizaje. REICE-Revista Electrónica Iberoamericana sobre Calidad, Eficacia y Cambio en Educación, 7 (1), 2-4. https://dialnet.unirioja.es/servlet/revista?codigo $=3322$

Bravo, M. \& Verdugo, S. (2007). Gestión escolar y éxito académico en condiciones de pobreza. REICE-Revista Electrónica Iberoamericana sobre Calidad, Eficacia y $\begin{array}{lllll}\text { Cambio en } & \text { Educación, } & 5 & \text { (1), } & \text { 121-144. }\end{array}$ https://dialnet.unirioja.es/servlet/revista?codigo $=3322$

Builes, M.E. (2016). El modelo pedagógico: Una estrategia para educar el mundo de hoy. Revista Reune, 1, 21-34. http://revistas.iue.edu.co/index.php/reune/issue/view/65

Builes, M. E., Díaz, J., Ramírez, G. (2014). Descripción de las prácticas educativas del grado décimo de la Institución Educativa Román Gómez: Una relación entre el modelo pedagógico institucional y las experiencias académicas de las estudiantes:

Citación del artículo: Díaz, J., Londoño-Vásquez, D. (2017). El reto de los directivos docentes: desarrollo integral a través de las prácticas educativas de la IE Román Gómez. Revista Katharsis, N 23, enero-julio 2017, pp.109-128, Disponible en http://revistas.iue.edu.co/index.php/katharsis 
Informe Técnico [material inédito]. Maestría en Educación y Desarrollo Humano, CINDE, Sabaneta.

Carrera H. C., Marín U. R. (2011). Modelo pedagógico para el desarrollo de competencias en educación Superior. Revista electrónica Actualidades Investigativas en Educación, 11 (1), pp. 1-32. http://www.redalyc.org/revista.oa?id=447

Cayulef, C. (2007). El liderazgo distribuido: una apuesta de dirección escolar de calidad. REICE-Revista Electrónica Iberoamericana sobre calidad, eficacia y cambio en educación 5 (5), 144-148. https://dialnet.unirioja.es/servlet/revista?codigo=3322

Colombia. Congreso de la República (1994). Ley general de educación, Ley 115. Ministerio de Educación Nacional, Bogotá.

Concha, C. (2007). Claves para la formación de directivos de instituciones Escolares. Revista Electrónica Iberoamericana sobre Calidad, Eficacia y Cambio en Educación, 5, (5), p.113-138.

Cruz, L. (2013). La autoridad legítima. México: Eón.

Delors, J. (1996). La educación encierra un tesoro. Madrid: Santillana ediciones.

Denzin, N. y Lincoln, I. (Coords.), Métodos de recolección y análisis de datos. Barcelona: Gedisa Editorial.

De Zubiría J., Ramirez, A., Ocampo, K., Marín, J. (2008). El modelo pedagógico predominante en Colombia [Tesis de Doctorado]. Instituto Merani, Bogotá. Recuperado de: http://www.institutomerani.edu.co/publicaciones/tesis/2008/tesis508.pdf.

Durkheim, E. (2000[1922]). Educación y sociología, 2. Ed. Barcelona: Península.

González B., D. M. (2010). Perspectivas del desarrollo humano. Medellín: Convenio Universidad de Manizales-CINDE.

Flórez, R. (1994). Hacia una pedagogía del conocimiento. Santafé de Bogotá: McGrawHill

Icfes (2011). Clasificación de las instituciones educativas. Disponible en http://www.icfesinteractivo.gov.co.

Londoño, D. (2015). Jóvenes y Literacidad: Un análisis sociolingüístico. Institución Universitaria de Envigado: Sello IUE.

Londoño, D., Castañeda, L.S. (2010). Diacronía del concepto juventud en las políticas colombianas. Katharsis, 10, 9-30.

Londoño, D., Castañeda, L.S. (2011). La comprensión como método en las ciencias sociales. Revista Virtual Católica del Norte, 31, 227-252. http://revistavirtual.ucn.edu.co/

Londoño, D., Ospina, H.F. (2016). La alfabetización crítica: requerimiento social. En: H.F. Ospina y C.A. Ramírez-López (ed). Pedagogía crítica latinoamericana y género. Bogotá: Siglo del Hombre Editores.

López, G., García, J., Slater, L. (2007). ¿Cómo resuelven los problemas los directores eficaces? Un estudio de directores de primaria mexicanos en su primer año de servicio. REICE-Revista Electrónica Iberoamericana sobre Calidad, Eficacia y Cambio en Educación, 5, (5), 139-143. https://dialnet.unirioja.es/servlet/revista?codigo=3322

López, J. N. E. (1996). Modernizacion curricular de las Instituciones educativas. Los PEI de cara al siglo XXI. Santa Fe de Bogotá: Editorial Libros y Libres.

Citación del artículo: Díaz, J., Londoño-Vásquez, D. (2017). El reto de los directivos docentes: desarrollo integral a través de las prácticas educativas de la IE Román Gómez. Revista Katharsis, N 23, enero-julio 2017, pp.109-128, Disponible en http://revistas.iue.edu.co/index.php/katharsis 
Linuesa, M.C. (2007). La complejidad de las relaciones teoría práctica en educación. Revista Teoría de la educacion 19. p. 25-46.Universidad de salamanca. http://campus.usal.es/ teoriadelaeducacion/Vol24_2_2012.html

Max-Neef, M., Elizalde, A., Hopenhayn, M. (1998). Desarrollo a escala humana: Conceptos, aplicaciones y algunas reflexiones. Montevideo: Editorial NordanComunidad.

Ñaupas, H., Mejía, E., Novoa, E., Villagómez, A. (2014). Metodología de la investigación cuantitativa - cualitativa y redacción de la tesis. Bogotá, Colombia: Ediciones de la $\mathrm{U}$.

Ocampo Flórez, E. (2010). Corrientes pedagógicas. Área de educación. Módulo 3 [material inédito]. CINDE - U de Manizales.

Pinto Blanco, A, M., Castro Quitora, L. (1999). Los modelos pedagógicos. Ibagué: Universidad del Tolima.

Roldán V. O. (2011). Los Proyectos Educativos: una posibilidad de desarrollo personal, institucional y comunitario. Sabaneta: Convenio Universidad de Manizales.

Sanz, C. T., Rodríguez, P. M. (2000). Tendencias pedagógicas en la realidad educativa actual. Colectivo de autores. Cepes Universidad de la Habana: Editorial universitaria, Tarija Bolivia.

Sen, A. (2001). El nivel de vida. Madrid: Editorial Complutense.

Sepúlveda, C., Lagomarsino, M. (2007). Estilos de gestión en educación municipalizada chilena y formación continua del profesorado. REICE-Revista Electrónica Iberoamericana sobre Calidad, Eficacia y Cambio en Educación, 5 (1). https://dialnet.unirioja.es/servlet/revista?codigo $=3322$

Tamés García, M. (2003). Educación, persona y sociedad Educación y Educadores, Bogotá: Universidad de La Sabana.

Vizcaíno Gutiérrez, L. (2010). Las funciones de la escuela. Revista digital Eduinnova, (26), 126-129, http://www.eduinnova.es/revista.html

Citación del artículo: Díaz, J., Londoño-Vásquez, D. (2017). El reto de los directivos docentes: desarrollo integral a través de las prácticas educativas de la IE Román Gómez. Revista Katharsis, N 23, enero-julio 2017, pp.109-128, Disponible en http://revistas.iue.edu.co/index.php/katharsis 